\title{
Risk Factors for and Clinical Outcomes of Polymicrobial Acinetobacter baumannii Bloodstream Infections
}

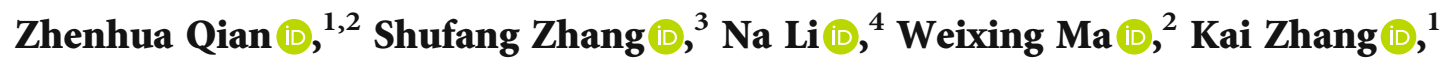 \\ Feizhen Song $\mathbb{D}^{1,5}$ Cheng Zheng $\mathbb{D}^{1,6}$ Li Zhong $\mathbb{D}^{1,7}$ Yesong Wang $\mathbb{D}^{1}$, Jiachang Cai $\mathbb{D}^{8}$, \\ Hongwei Zhou $\mathbb{D}^{8},{ }^{8}$ Wei Cui $\mathbb{D}^{1},{ }^{1}$ and Gensheng Zhang $\mathbb{D}^{1}$ \\ ${ }^{1}$ Department of Critical Care Medicine, Second Affiliated Hospital, Zhejiang University School of Medicine, Hangzhou, \\ Zhejiang 310009, China \\ ${ }^{2}$ Department of Critical Care Medicine, Shaoxing Central Hospital, Shaoxing, Zhejiang 312000, China \\ ${ }^{3}$ Department of Cardiology, Second Affiliated Hospital, Zhejiang University School of Medicine, Hangzhou, Zhejiang 310009, China \\ ${ }^{4}$ Department of Respiratory Medicine, The First Hospital of Jiaxing (The Affiliated Hospital of Jiaxing University), Jiaxing, \\ Zhejiang 314001, China \\ ${ }^{5}$ Department of Critical Care Medicine, Shengzhou People's Hospital, Shaoxing, Zhejiang 312000, China \\ ${ }^{6}$ Department of Critical Care Medicine, Taizhou Municipal Hospital, Taizhou, Zhejiang 318000, China \\ ${ }^{7}$ Department of Critical Care Medicine, Huzhou First People's Hospital, Huzhou, Zhejiang 312000, China \\ ${ }^{8}$ Clinical Microbiology Laboratory, Second Affiliated Hospital, Zhejiang University School of Medicine, Hangzhou 310009, China
}

Correspondence should be addressed to Gensheng Zhang; genshengzhang@zju.edu.cn

Received 23 September 2021; Revised 25 January 2022; Accepted 1 February 2022; Published 27 February 2022

Academic Editor: Haruki Komatsu

Copyright (C) 2022 Zhenhua Qian et al. This is an open access article distributed under the Creative Commons Attribution License, which permits unrestricted use, distribution, and reproduction in any medium, provided the original work is properly cited.

Background. Although the clinical features of Acinetobacter baumannii bloodstream infection are well described, the specific clinical characteristics of polymicrobial Acinetobacter baumannii bloodstream infection have been rarely reported. The objective of this study was to examine the risk factors for and clinical outcomes of polymicrobial Acinetobacter baumannii bloodstream infection. Methods. A retrospective observational study was performed from January 2013 to December 2018 in a tertiary hospital. All patients with Acinetobacter baumannii bloodstream infection were enrolled, and the data were collected from the electronic medical records. Results. A total of 594 patients were included, 21\% (126/594) of whom had polymicrobial infection. The most common copathogen was Klebsiella pneumoniae (20.81\%), followed by Pseudomonas aeruginosa (16.78\%) and Enterococcus faecium (12.08\%). Compared with monomicrobial Acinetobacter baumannii bloodstream infection, polymicrobial Acinetobacter baumannii bloodstream infection mostly originated from the skin and soft tissue $(28.6 \%$ vs. $10.5 \%$, $p<0.001)$. Multivariate analysis revealed that burn injury was independently associated with polymicrobial Acinetobacter baumannii bloodstream infection (adjusted odds ratio, 3.569; 95\% confidence interval, 1.954-6.516). Patients with polymicrobial Acinetobacter baumannii bloodstream infection were more likely to have a longer hospital length of stay [40 (21, $68)$ vs. $27(16,45), p<0.001]$ and more hospitalization days after bloodstream infection than those with monomicrobial Acinetobacter baumannii bloodstream infection $[22(8,50)$ vs. $13(4,28), p<0.001]$. However, no significant difference in mortality was observed between the two groups. Conclusions. Approximately one-fifth of Acinetobacter baumannii bloodstream infections were polymicrobial in this cohort. The main sources were skin and soft tissue infections, and burn injury was the only independent risk factor. Although mortality did not differ between the groups, considering the limitations of the study, further studies are required to assess the impact of polymicrobial (vs. monomicrobial) Acinetobacter baumannii bloodstream infection on outcomes. 


\section{Background}

Bloodstream infection (BSI) is a major cause of hospitalacquired sepsis and causes approximately 157,000 deaths per year in Europe and more than 79,000 deaths per year in North America [1]. As an important gram-negative bacterium, Acinetobacter baumannii (AB) accounts for 9\% 35\% of all BSIs, with an increasing tendency [2, 3]. Due to increases in antibiotic exposure, invasive operations, and carbapenem resistance, $\mathrm{AB}$ has become the most common cause of BSI in critically ill patients [4-6]. The overall mortality rate in patients with AB-BSI ranges widely from $29 \%$ to $63 \%$ [7-9]. Therefore, AB-BSI has become a major challenge in the clinic due to the rapid spread of multidrug resistance among $\mathrm{AB}$ and its high associated morbidity and mortality [9-12].

Most BSIs are monomicrobial, but the trend of polymicrobial BSIs is increasing $[13,14]$. According to a recent systematic review and meta-analysis, approximately a quarter (range $0 \%$ to $>60 \%$, depending on the setting and definition) of AB-BSIs are polymicrobial [15]. Although a higher Acute Physiology and Chronic Health Evaluation II (APACHE II) score and a higher frequency of severe sepsis/septic shock are more often observed in patients with polymicrobial BSI than in those with monomicrobial BSI $[16,17]$, the difference in the mortality rate between the two populations is controversial $[15,18-20]$. Limitations of the available literature include the following: (1) polymicrobial AB-BSI is commonly excluded [15], and (2) available data on the impact of polymicrobial (vs. monomicrobial) AB-BSI on clinical outcomes are conflicting [15]. Furthermore, only one previous study has evaluated the impact of polymicrobial AB-BSI on clinical outcomes [21]; thus, comparisons of the characteristics of and risk factors for polymicrobial vs. monomicrobial infections are lacking [15].

The aim of this retrospective study was to determine differences in the proportions and risk factors for and outcomes of polymicrobial and monomicrobial AB-BSIs.

\section{Materials And Methods}

2.1. Patients and Study Design. We reviewed the medical records of patients who were admitted to the Second Affiliated Hospital, Zhejiang University School of Medicine, a 3200-bed tertiary healthcare facility in Hangzhou, China, between January 2013 and December 2018. The present study received human research ethics approval (No. 2019116) from the Ethics Committee of the Second Affiliated Hospital, Zhejiang University School of Medicine. Due to the retrospective nature of the study, the Ethics Committee determined that no patient consent was required.

We collected all AB-positive blood culture results. At least two blood culture samples were collected (at the same time but from different sites) from each patient at the time of the event. Only the first episode was included for patients with more than one episode of AB-BSI. The exclusion criteria were as follows: (a) age < 18 years old; (b) classification of $\mathrm{AB}$ as a nonpathogenic bacterium; or (c) incomplete or missing case data. Common skin contaminants (e.g., Bacillus spp., Corynebacterium spp., Micrococcus spp., streptococci, Lactobacillus spp., and coagulase-negative staphylococci) were considered pathogens only when they were present in two or more consecutive blood cultures from separate blood draws [22]. If skin contaminants were coisolated with $A B$ from a single blood culture, such infections were classified as monomicrobial.

2.2. Data Collection. Documented patient demographics, including age, sex, and dates of hospital admission and discharge, were collected. Medical history data included comorbidities at admission, prior hospitalization before BSI, major surgery, the presence of invasive devices (such as central venous catheters, urinary catheters, or drainage catheters), mechanical ventilation, and haemodialysis within 1 week before BSI onset were reviewed. The following parameters within $24 \mathrm{~h}$ before BSI onset, reflecting the severity of disease, were collected: the sequential organ failure assessment (SOFA) score, Pitt bacteraemia score, Charlson Comorbidity Index (CCI) score, and Acute Physiology and Chronic Health Evaluation (APACHE) II score. In the first $24 \mathrm{~h}$ following the onset of BSI, inflammatory markers such as white blood cell count, procalcitonin level, C-reactive protein level, and liver and kidney function indicators were measured. Microbiological data, such as species involved in polymicrobial AB-BSI, the likely source of the BSI, and sensitivity to antibiotics were gathered. Primary outcomes (all-cause 14day and 28-day in-hospital mortality) and secondary outcomes (length of total hospital stay and length of hospital stay after BSI onset) were also collected.

\subsection{Species Identification and Antibiotic Sensitivity Test.} Blood culture was performed by using a BacT/ALERT 3D system (Becton-Dickinson, Sparks, MD, USA) in the microbiology laboratory. Species identification was completed using Bruker Daltonics data analysis software. Antibiotic susceptibility was determined with a VITEK 2 system (Card number: AST-GN16; AST-GP67) or the Kirby-Bauer disk diffusion method (Oxford, UK) according to the recommendations of the Clinical and Laboratory Standards Institute (CLSI) [23]. During the study period, there were no changes in microbiological laboratory techniques.

2.4. Definitions. AB-BSI was defined as at least one blood culture positive for $\mathrm{AB}$ accompanied by two or more of the following symptoms and signs of infection: fever, hypothermia, tachypnoea, tachycardia, leucocytosis, or leukopenia or other corresponding clinical symptoms and signs and the exclusion of specimen contamination $[21,22,24]$. The onset of AB-BSI was defined as the time when the positive blood culture sample was obtained. Polymicrobial AB-BSI was defined as the isolation of $\mathrm{AB}$ and one or more other microorganism from the same blood culture, excluding contamination $[21,25]$. The primary infection source of BSI was determined according to the definitions of the Center for Disease Control (CDC) [22]. Nosocomial BSI was defined as the first positive blood culture obtained $\geq 48 \mathrm{~h}$ after hospital admission, with no evidence of infection at admission [7, 14]. Prior exposure to antimicrobial agents was defined as 


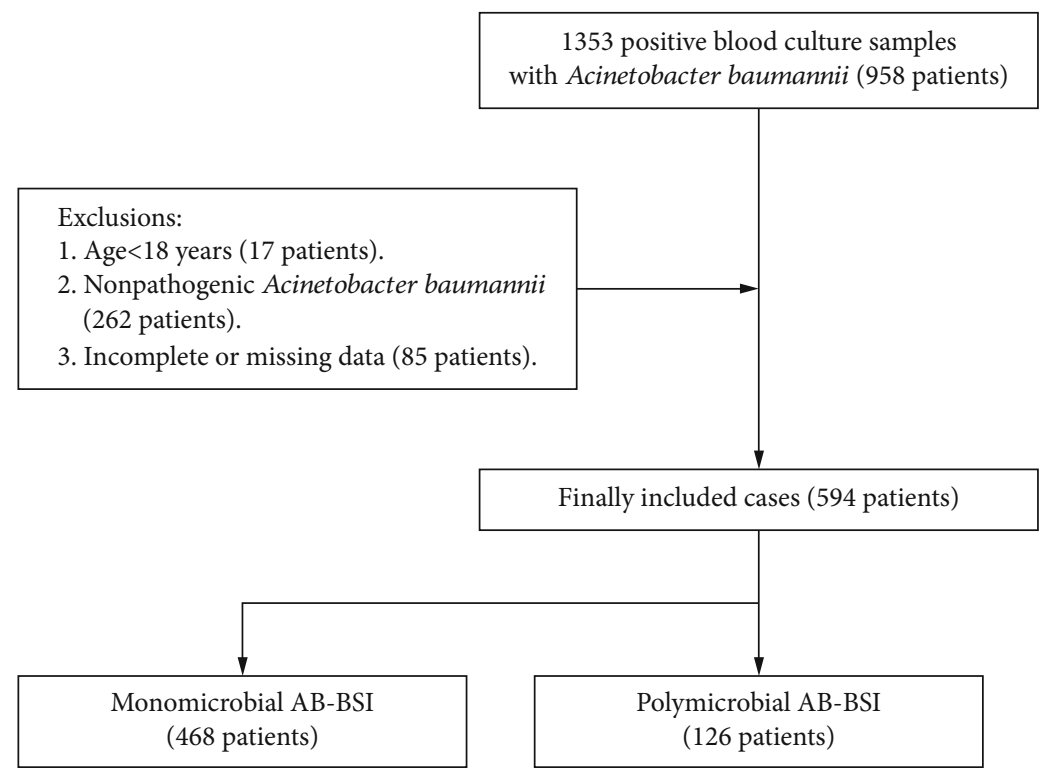

FIGURE 1: Flowchart of study participant enrollment. Abbreviations: AB-BSI: Acinetobacter baumannii bloodstream infection.

antimicrobial treatment for at least $72 \mathrm{~h}$ within 30 days prior to the positive blood culture [26]. Appropriate empiric antimicrobial treatment was defined as at least one antibiotic that was active against the pathogenic microorganisms, as confirmed by an in vitro sensitivity test within $48 \mathrm{~h}$ of BSI onset [9]. Multidrug resistance was defined as $\mathrm{AB}$ resistance to $\geq 3$ classes of antibiotics (quinolones, extended-spectrum cephalosporins, $\beta$-lactam $/ \beta$-lactamase inhibitor combinations, aminoglycosides, and carbapenems) [27]. Sepsis and septic shock were defined according to the definition of the International Sepsis Definitions Conference [24].

2.5. Statistical Analysis. Statistical analysis was performed using SPSS 23.0 software (IBM Corp, Armonk, NY, USA). Continuous variables are presented as means \pm standard deviations if normally distributed and as medians and interquartile ranges (IQRs) if nonnormally distributed. Categorical variables were compared by the Pearson $\chi^{2}$ test or Fisher's exact test. Variables with a $p$ value $<0.2$ in the univariate logistic regression analysis were entered into the multivariate logistic regression model to determine the independent variables. In addition, clinical scores, such as the APACHE II score, SOFA score, Pitt bacteraemia score, and CCI score, were examined in the multivariate logistic regression model. We excluded patients without intensive care unit (ICU) admission in the analysis of total ICU residence days and ICU residence days after BSI. All tests were 2 -tailed, and $p<0.05$ was considered significant.

\section{Results}

3.1. Demographic Characteristics. From January 2013 to December 2018, a total of 1353 blood culture samples positive for $A B$ were initially identified, and 594 patients with AB-BSI were recruited for analysis. A total of $126(21 \%)$ cases were polymicrobial AB-BSI, and 468 (79\%) cases were monomicrobial AB-BSI (Figure 1).
The median patient age was 61 years (IQR, 48-71), and $70.5 \%$ were male. The patients with monomicrobial ABBSI were older than those with polymicrobial AB-BSI (age $\geq 60$ years, $54.5 \%$ vs. $44.4 \%, p<0.05$ ). A total of $96.1 \%$ of the patients (571/594) had at least one comorbidity, and a significantly higher rate of burn injury was observed in the polymicrobial $\mathrm{AB}$-BSI group than in the monomicrobial AB-BSI group $(23.0 \%$ vs. $8.3 \%, p<0.05)$. The detailed demographic characteristics of the patients are shown in Table 1.

3.2. Biological Indicators. Comparisons of biological indicators between the polymicrobial AB-BSI and monomicrobial AB-BSI groups are shown in Table 2. The glutamicoxaloacetic transaminase (GOT) level was higher in the polymicrobial AB-BSI group, but there were no significant differences in other liver function indicators or biochemical indicators between the two groups.

3.3. Independent Risk Factors for Polymicrobial AB-BSI. Table 3 shows the results of the multivariate logistic regression analysis. Burn injury was the only independent risk factor for polymicrobial AB-BSI (adjusted odds ratio [aOR], 3.569; 95\% confidence interval [CI], 1.954-6.516).

3.4. Aetiologic Agents of BSIs. In addition to AB, 149 microorganisms were isolated from 126 patients with polymicrobial AB-BSI, with two microorganisms accounting for $84.1 \%(106 / 126)$ and three microorganisms accounting for $15.87 \%$ (20/126). Gram-negative bacteria, gram-positive bacteria, and fungi accounted for $67.1 \%, 30.2 \%$, and $2.7 \%$ of polymicrobial AB-BSI infections, respectively. The most common bacterium involved in polymicrobial AB-BSIs was Klebsiella pneumoniae $(31 / 149,20.8 \%)$, followed by Pseudomonas aeruginosa $(25 / 149,16.8 \%)$ and Enterococcus faecium $(18 / 149,12.1 \%)$. A detailed description of the isolated microorganisms is shown in Figure 2. 
TABLE 1: Demographic and clinical characteristics of monomicrobial AB-BSI and polymicrobial AB-BSI patients.

\begin{tabular}{|c|c|c|c|c|}
\hline Characteristics & Total $(n=594)$ & Monomicrobial $(n=468)$ & Polymicrobial $(n=126)$ & $p$ value \\
\hline Age $\geq 60$ years & $311(52.4 \%)$ & $255(54.5 \%)$ & $56(44.4 \%)$ & 0.046 \\
\hline Male sex & $419(70.5 \%)$ & $330(70.5 \%)$ & $89(70.6 \%)$ & 0.979 \\
\hline \multicolumn{5}{|l|}{ Comorbidities } \\
\hline Diabetes mellitus & $82(13.8 \%)$ & $62(13.2 \%)$ & $20(15.9 \%)$ & 0.448 \\
\hline Chronic kidney disease & $67(11.3 \%)$ & $51(10.9 \%)$ & $16(12.7 \%)$ & 0.571 \\
\hline Chronic liver disease & $45(7.6 \%)$ & $34(7.3 \%)$ & $11(8.7 \%)$ & 0.581 \\
\hline $\mathrm{COPD}^{\mathrm{a}}$ or severe asthma & $77(13 \%)$ & $59(12.6 \%)$ & $18(14.3 \%)$ & 0.618 \\
\hline Chronic cardiac insufficiency & $130(21.9 \%)$ & $98(20.9 \%)$ & $32(25.4 \%)$ & 0.283 \\
\hline Solid tumour & $77(13 \%)$ & $68(14.5 \%)$ & $9(7.1 \%)$ & 0.028 \\
\hline Trauma & $146(24.6 \%)$ & $119(25.4 \%)$ & $27(21.4 \%)$ & 0.355 \\
\hline Burn injury & $68(11.4 \%)$ & $39(8.3 \%)$ & $29(23.0 \%)$ & $<0.001$ \\
\hline Hypertension & $190(32 \%)$ & $145(31 \%)$ & $45(35.7 \%)$ & 0.312 \\
\hline Cerebrovascular accident & $166(27.9 \%)$ & $131(28 \%)$ & $35(27.8 \%)$ & 0.962 \\
\hline $\mathrm{CCI}^{\mathrm{b}}$ score, median (IQR) & $2(1,3)$ & $2(1,3)$ & $2(0,3)$ & 0.507 \\
\hline APACHE II score, median & $18(13,22)$ & $18(14,22)$ & $17(13,22)$ & 0.375 \\
\hline SOFA score, median & $6(4,9)$ & $6(4,9)$ & $6(3,9)$ & 0.442 \\
\hline Pitt bacteraemia score, median & $4(3,6)$ & $4(3,6)$ & $4.5(3,6)$ & 0.704 \\
\hline \multicolumn{5}{|l|}{ Hospitalization ward } \\
\hline Medical & $8(1.3 \%)$ & $5(1.1 \%)$ & $3(2.4 \%)$ & 0.257 \\
\hline Surgical & $63(10.6 \%)$ & $47(10 \%)$ & $16(12.7 \%)$ & 0.390 \\
\hline ICU & $523(88 \%)$ & $416(88.9 \%)$ & $107(84.9 \%)$ & 0.223 \\
\hline \multicolumn{5}{|l|}{ Previous treatment } \\
\hline Hyperalimentation & $227(38.2 \%)$ & $176(37.6 \%)$ & $51(40.5 \%)$ & 0.556 \\
\hline Mechanical ventilation $(n)$ & $550(92.6 \%)$ & $434(92.7 \%)$ & $116(92.1 \%)$ & 0.798 \\
\hline Surgery & $369(62.1 \%)$ & $295(63 \%)$ & $74(58.7 \%)$ & 0.377 \\
\hline Blood transfusion & $247(41.6 \%)$ & $189(40.4 \%)$ & $58(46.0 \%)$ & 0.254 \\
\hline Renal replacement therapy & $109(18.4 \%)$ & $84(17.9 \%)$ & $25(19.8 \%)$ & 0.626 \\
\hline Carbapenem exposure & $237(39.9 \%)$ & $182(38.9 \%)$ & $55(43.7 \%)$ & 0.333 \\
\hline \multicolumn{5}{|l|}{ Invasive devices } \\
\hline Central line & $558(93.9 \%)$ & $441(94.2 \%)$ & $117(92.9 \%)$ & 0.566 \\
\hline Indwelling urinary catheter & $541(91.1 \%)$ & $424(90.6 \%)$ & $117(92.9 \%)$ & 0.430 \\
\hline Drainage (any site) & $253(42.6 \%)$ & $197(42.1 \%)$ & $56(44.4 \%)$ & 0.636 \\
\hline Prior hospital stay, median days (IQR) & $11(7,18)$ & $11(6,18)$ & $11.5(7,20)$ & 0.367 \\
\hline Nosocomial infection & $559(94.1 \%)$ & $438(93.6 \%)$ & $121(96 \%)$ & 0.301 \\
\hline
\end{tabular}

${ }^{\mathrm{a}}$ Chronic obstructive pulmonary disorder. ${ }^{\mathrm{b}}$ Charlson Comorbidity Index.

The source of AB-BSI was mainly respiratory tract infection $(25.9 \%, 154 / 594)$, followed by primary BSI $(22.4 \%, 133 /$ $594)$ and central venous catheter-related infection $(15 \%, 89 /$ 451). Compared with those of monomicrobial AB-BSI, the sources of polymicrobial AB-BSI were more frequently skin and soft tissue infections $(28.6 \%$ vs. $10.5 \%, p<0.001)$ and less frequently respiratory tract $(15.1 \%$ vs. $28.8 \%, p<0.05)$ and intracranial infections $(2.4 \%$ vs. $8.5 \%, p=0.018)$ (Table 4).

3.5. Antibiotic Resistance and Appropriate Therapy. All BSIs were treated with antibiotics. Antibiotic sensitivity testing showed that there was no difference in sensitivity between the monomicrobial AB-BSI and polymicrobial AB-BSI groups. The resistance rates of $\mathrm{AB}$ to ciprofloxacin, ceftazidime, nitrofurantoin, and carbapenems were very high in both groups (more than 90\%). In contrary, the ratios of resistance of $\mathrm{AB}$ to amikacin, tigecycline, and colistin were relatively low in the two groups (less than 30\%). Nine strains of $\mathrm{AB}$ resistant to colistin were found in the monomicrobial AB-BSI group, while none was found in the polymicrobial AB-BSI group (Table 4).

A total of $28.5 \%(169 / 594)$ of the patients received appropriate empiric antibiotic therapy. Notably, the rate of appropriate empirical antimicrobial therapy in patients with polymicrobial AB-BSI was substantially higher than that in patients with monomicrobial AB-BSI (38.9\% vs. $25.6 \%, p<$ $0.05)$. 
TABLE 2: Comparison of biological indicators between the monomicrobial AB-BSI and polymicrobial AB-BSI groups.

\begin{tabular}{|c|c|c|c|c|}
\hline Biochemical indicators & Total $(n=594)$ & Monomicrobial $(n=468)$ & Polymicrobial $(n=126)$ & $p$ value \\
\hline Temperature $\left({ }^{\circ} \mathrm{C}\right)(\mathrm{IQR})$ & $38.6(38.0,39.1)$ & $38.6(38.1,39.1)$ & $38.5(38.0,39.03)$ & 0.139 \\
\hline $\mathrm{WBC}^{\mathrm{a}}\left(\times 10^{9} / \mathrm{L}\right)(\mathrm{IQR})$ & $10.2(6.9,14.3)$ & $10.6(7.03,14.4)$ & $9.65(6.65,13.68)$ & 0.354 \\
\hline Haematocrit (\%) (IQR) & $25.15(21.7,29.93)$ & $25.3(22.0,30.48)$ & $24.2(21.0,28.33)$ & 0.031 \\
\hline Platelets $\left(\times 10^{9} / \mathrm{L}\right)(\mathrm{IQR})$ & $144(77.75,228.25)$ & $147.5(79,234.75)$ & $128.5(69.75,217.5)$ & 0.340 \\
\hline $\mathrm{ANC}^{\mathrm{b}}$ (IQR) & $8.71(5.76,12.77)$ & $9.02(5.87,12.93)$ & $8.35(5.27,12.10)$ & 0.276 \\
\hline $\operatorname{Albumin}(\mathrm{g} / \mathrm{L})($ mean $\pm \mathrm{SD})$ & $30.65 \pm 5.82$ & $30.85 \pm 5.82$ & $30.4 \pm 5.79$ & 0.201 \\
\hline $\mathrm{GPT}^{\mathrm{c}}(\mathrm{U} / \mathrm{L})(\mathrm{IQR})$ & $37(23,68)$ & $37(23,70)$ & $39(27.5,63.25)$ & 0.330 \\
\hline $\mathrm{GOT}^{\mathrm{d}}(\mathrm{U} / \mathrm{L})(\mathrm{IQR})$ & $41(26,67)$ & $39(26,66)$ & $48.5(28,75.5)$ & 0.021 \\
\hline $\mathrm{TBil}^{\mathrm{e}}$ (umol/L) (IQR) & $19.6(12.18,34.23)$ & $19.6(12.13,35.78)$ & $19.65(12.18,31.95)$ & 0.787 \\
\hline $\mathrm{SCr}^{\mathrm{f}}$ (umol/L) (IQR) & $62(46,99)$ & $63(46,100.75)$ & $57(44.75,95.25)$ & 0.673 \\
\hline Lactic acid (mol/L) (IQR) & $2.1(1.3,3.1)$ & $2.1(1.3,3.2)$ & $2(1.28,2.83)$ & 0.461 \\
\hline $\mathrm{CRP}^{\mathrm{g}}(\mathrm{mg} / \mathrm{L})(\mathrm{IQR})$ & $119.85(67.05,195.5)$ & $120.25(67.90,199.75)$ & $119.35(63.98,180.0)$ & 0.431 \\
\hline $\mathrm{PCT}^{\mathrm{h}}(\mathrm{ng} / \mathrm{mL})(\mathrm{IQR})$ & $1.74(0.45,6.53)$ & $1.72(0.44,6.71)$ & $1.91(0.50,6.31)$ & 0.979 \\
\hline
\end{tabular}

${ }^{\mathrm{a}}$ White blood count. ${ }^{\mathrm{b}}$ Absolute neutrophil count. ${ }^{\mathrm{c}}$ Glutamic-pyruvic transaminase. ${ }^{\mathrm{d}}$ Glutamic-oxaloacetic transaminase. ${ }^{\mathrm{e}}$ Total bilirubin. ${ }^{\mathrm{f}}$ Serum creatinine. ${ }^{\mathrm{g}} \mathrm{C}$-reactive protein. ${ }^{\mathrm{h}}$ Procalcitonin.

3.6. Outcomes. The prevalence of septic shock was similar (25.4\% vs. $28.8 \%, p=0.445)$ between the two groups. Patients with monomicrobial AB-BSI had more total ICU residence days $[25(14,49)$ vs. $20(12,32), p<0.05]$ and ICU residence days after BSI $[11(3,33)$ vs. $8(3,18), p<$ 0.05] than those with polymicrobial AB-BSI, while those with polymicrobial AB-BSI had a higher 14-day survival rate (Figure 3 ) and more total hospitalization days $[40(21,68)$ vs. $27(16,45), p<0.001]$ and hospitalization days after BSI onset $[22(8,50)$ vs. $13(4,28), p<0.001]$. However, there were no significant differences in the 14-day and 28day in-hospital mortality rates between the two groups (Table 5).

\section{Discussion}

The main findings of our study are as follows: (1) a high proportion of AB-BSIs were polymicrobial; (2) gram-negative bacteria (especially Klebsiella pneumoniae and Pseudomonas aeruginosa) were the most common copathogens, followed by gram-positive bacteria (especially Staphylococcus aureus and Enterococcus spp.) and fungi; (3) younger age, burn injury, and skin and soft tissue sources of the bacteraemia were associated with polymicrobial (vs. monomicrobial) $\mathrm{AB}-\mathrm{BSI}$, and burn injury was the only independent risk factor for polymicrobial AB-BSI; and (4) polymicrobial AB-BSI was associated with longer total and post-BSI hospital stays, but associated mortality rates did not differ significantly.

In the current study, $21 \%$ of AB-BSIs were polymicrobial. The range of the proportion of polymicrobial AB-BSI varies widely in the literature (ranging from 0 to $>60 \%$ ), but the estimated pooled proportion in a recent metaanalysis was very close to our findings [15]. Excluding common skin contaminants, gram-negative bacteria were the most common copathogens in polymicrobial AB-BSIs, consistent with the previous findings of the majority of BSIs caused by Pseudomonas aeruginosa, Klebsiella pneumoniae, and Staphylococcus aureus [17, 28, 29]. Similarly, the most common copathogens in mixed-enterococcal BSIs reported in our previous study were gram-negative bacteria $(57.1 \%)$, followed by gram-positive bacteria (38.3\%) and fungi (4.6\%) [18]. These results suggest that gram-negative bacteria are the most frequent agents of BSIs and polymicrobial BSIs.

Contrary to other studies $[21,25,30]$, there was no difference in the outcome between BSI groups despite a $13.3 \%$ higher rate of appropriate empiric antimicrobial treatment in patients with polymicrobial AB-BSI (38.9\% vs. $25.6 \%, p<0.05)$. The actual rates of appropriate empiric antimicrobial treatment in both groups were low, as both were less than $40 \%$, which might have had a relatively weak impact on the outcomes in patients in both groups. Indeed, rapid pathogen identification and antimicrobial susceptibility testing are crucial for targeted therapy and the initiation of appropriate therapy [2, 31]. However, conventional microbiological processing of a blood culture requires 48$72 \mathrm{~h}$. Molecular rapid diagnostic testing (RDT) has been developed to optimize initial empiric antimicrobial therapy. Nucleic acid amplification tests (NAATs) can identify pathogens approximately $6 \mathrm{~h}$ after a blood sample is drawn, and the $\mathrm{T} 2$ magnetic resonance (T2MR) assay is a relatively new, fully automated method that can directly detect a range of pathogens in whole blood [32]. However, their clinical performance remains to be validated. RDT plays a prominent role in special pathogen detection, especially in the monitoring of multidrug-resistant gram-negative bacteria [32]. The Amplex eazyplex SuperBug Acineto test can quickly identify carbapenem-resistant $\mathrm{AB}$ (CRAB) BSI, and the rapid and simple setup, requiring minimal hands-on time, makes this test suitable for implementation in settings with a high prevalence of CRAB [33]. In addition to conventional blood culture, other rapid diagnostic tools for the detection of AB-BSI should be considered in clinical practice.

In this study, patients with monomicrobial AB-BSI were older and had more tumours than those with polymicrobial AB-BSI. However, the CCI score, APACHE II score, and 
TABLE 3: Logistic regression analysis of factors associated with polymicrobial AB-BSI.

\begin{tabular}{|c|c|c|c|c|}
\hline \multirow{2}{*}{ Characteristics } & \multicolumn{2}{|c|}{ Univariable analysis } & \multicolumn{2}{|c|}{ Multivariable analysis } \\
\hline & Unadjusted OR (95\% CI) & $p$ value & Adjusted OR (95\% CI) & $p$ value \\
\hline Age $\geq 60$ years & $0.668(0.450,0.993)$ & 0.046 & $0.855(0.546,1.338)$ & 0.493 \\
\hline Male sex & $0.994(0.646,1.531)$ & 0.979 & & \\
\hline Diabetes mellitus & $1.236(0.715,2.136)$ & 0.449 & & \\
\hline Chronic kidney disease & $1.189(0.653,2.166)$ & 0.571 & & \\
\hline Chronic liver disease & $1.211(0.600,2.484)$ & 0.582 & & \\
\hline $\mathrm{COPD}^{\mathrm{a}}$ or severe asthma & $0.866(0.490,1.529)$ & 0.619 & & \\
\hline Chronic cardiac insufficiency & $0.779(0.492,1.231)$ & 0.284 & & \\
\hline Solid tumour & $1.936(1.027,3.651)$ & 0.028 & $2.120(0.973,4.620)$ & 0.059 \\
\hline Trauma & $1.250(0.779,2.008)$ & 0.355 & & \\
\hline Burn injury & $2.313(1.664,3.214)$ & $<0.001$ & $3.569(1.954,6.516)$ & $<0.001$ \\
\hline Hypertension & $0.808(0.534,1.222)$ & 0.313 & & \\
\hline Cerebrovascular accident & $1.011(0.652,1.568)$ & 0.962 & & \\
\hline $\mathrm{CCI}^{\mathrm{b}}$ & $0.965(0.869,1.071)$ & 0.500 & $1.119(0.984,1.274)$ & 0.087 \\
\hline APACHE II score & $0.986(0.956,1.016)$ & 0.352 & $0.980(0.934,1.029)$ & 0.423 \\
\hline SOFA score & $0.991(0.942,1.042)$ & 0.721 & $0.986(0.925,1.052)$ & 0.671 \\
\hline Pitt bacteraemia score & $1.017(0.936,1.104)$ & 0.696 & $1.083(0.969,1.212)$ & 0.162 \\
\hline Medical & $0.443(0.104,1.878)$ & 0.269 & & \\
\hline Surgical & $0.768(0.419,1.405)$ & 0.391 & & \\
\hline ICU & $1.421(0.806,2.504)$ & 0.225 & & \\
\hline Hyperalimentation & $0.886(0.593,1.325)$ & 0.556 & & \\
\hline Mechanical ventilation $(n)$ & $1.100(0.528,2.293)$ & 0.798 & & \\
\hline Surgery & $1.198(0.802,1.790)$ & 0.377 & & \\
\hline Blood transfusion & $0.794(0.535,1.180)$ & 0.254 & & \\
\hline Renal replacement therapy & $0.884(0.537,1.453)$ & 0.626 & & \\
\hline Carbapenem exposure & $0.821(0.552,1.223)$ & 0.333 & & \\
\hline Central line & $1.256(0.575,2.745)$ & 0.567 & & \\
\hline Indwelling urinary catheter & $0.741(0.352,1.562)$ & 0.431 & & \\
\hline Drainage (any site) & $0.909(0.611,1.351)$ & 0.636 & & \\
\hline Prior hospital stay & $1.005(0.996,1.015)$ & 0.293 & & \\
\hline Nosocomial infection & $0.603(0.229,1.588)$ & 0.306 & & \\
\hline
\end{tabular}

${ }^{\mathrm{a}}$ Chronic obstructive pulmonary disorder. ${ }^{\mathrm{b}}$ Charlson Comorbidity Index.

SOFA score, reflecting the severity of underlying disease, were not different between the groups, similar to the results of a recent study [21]. The only independent risk factor for polymicrobial AB-BSI according to the multivariate analysis was burn injury. Forty-three percent (29/68) of burn patients developed polymicrobial AB-BSI, which was consistent with the results of Tang et al.'s study, which reported that more than $20 \%$ of burn patients developed polymicrobial BSI [34]. Burn injury as an independent risk factor for polymicrobial AB-BSI in the current study might partially reflect the significantly higher rates of skin and soft tissue infections as infection sources in the polymicrobial AB-BSI group than in the monomicrobial AB-BSI group (28.6\% vs. $10.5 \%, p<0.05)$. As described in the previous studies, burn injury can cause the downregulation of cellular and humoral immune responses, extensive disruption of the skin barrier, and gastrointestinal bacterial translocation, resulting in prolonged hospitalization and the need for invasive diagnostic/ therapeutic management [35-37]; thus, burn patients have a high risk of BSI. Even though many pathogens colonize the skin, these bacteria are more likely to invade the blood in burn patients, causing polymicrobial BSI, such as polymicrobial AB-BSI in the current study.

The available literature is controversial with regard to the impact of polymicrobial (vs. monomicrobial) AB-BSI on mortality, with most studies showing nonsignificant differences and few studies showing significantly higher or significantly lower mortality rates in polymicrobial AB-BSI patients [15]. According to a recent meta-analysis, polymicrobial AB-BSI was associated with a lower 28-day (but not in-hospital) mortality rate than monomicrobial ABBSI, which is in agreement with the findings of our study, prompting the hypothesis that other (usually more susceptible) coisolates are the primary pathogens and $\mathrm{AB}$ is a secondary pathogen [15]. The larger study sample in the meta-analysis allowed sufficient statistical power to 


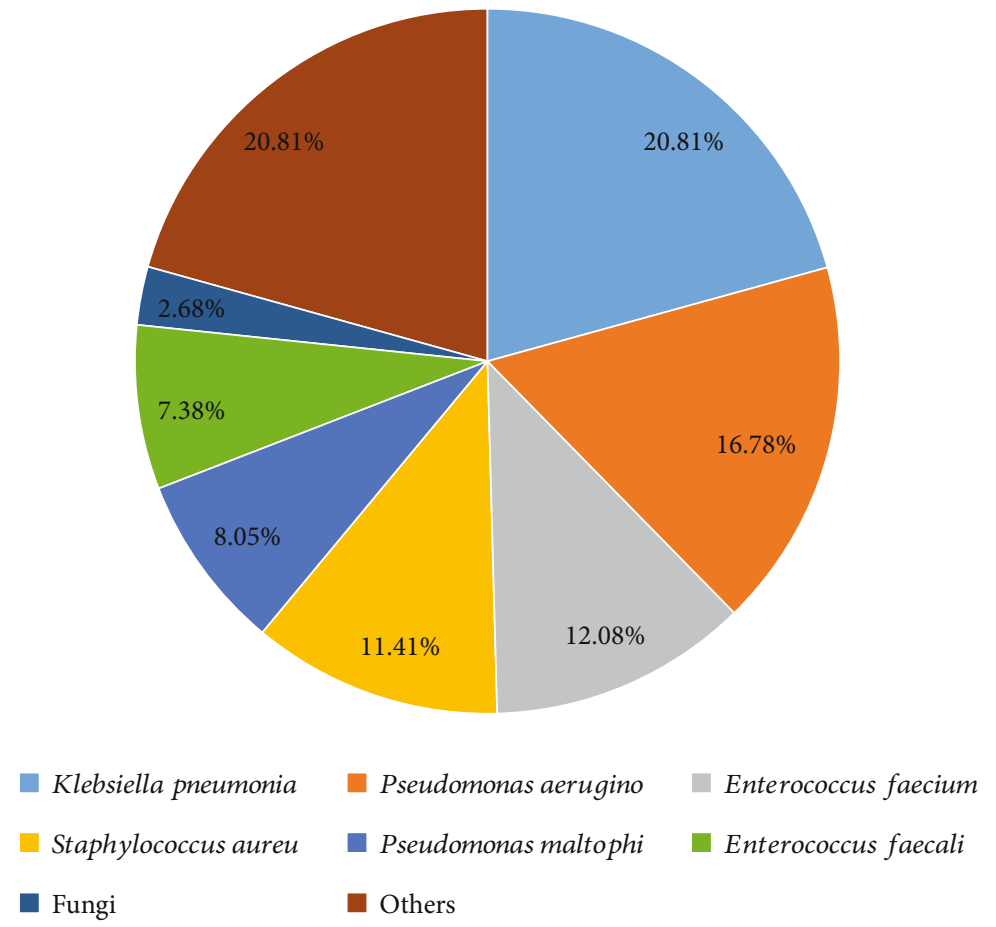

FIGURE 2: Distributions of coinfecting organisms in polymicrobial Acinetobacter baumannii bloodstream infections. Fungi: Candida albicans, Candida near smooth, and Candida tropicalis; others: Escherichia coli, Proteus mirabilis, Enterobacter cloacae, Serratia marcescens, etc.; Abbreviations: AB-BSI: Acinetobacter baumannii bloodstream infection.

TABLE 4: Comparisons of the microbiological characteristics of monomicrobial AB-BSIs and polymicrobial AB-BSIs.

\begin{tabular}{|c|c|c|c|c|}
\hline Antibiotic resistance & Total $(n=594)$ & Monomicrobial $(n=468)$ & Polymicrobial $(n=126)$ & $p$ value \\
\hline \multicolumn{5}{|l|}{ Source of BSI } \\
\hline Respiratory tract & $154(25.9 \%)$ & $135(28.8 \%)$ & $19(15.1 \%)$ & 0.002 \\
\hline Central venous catheter & $89(15 \%)$ & $64(13.7 \%)$ & $25(19.8 \%)$ & 0.085 \\
\hline Skin and soft tissue & $85(14.3 \%)$ & $49(10.5 \%)$ & $36(28.6 \%)$ & $<0.001$ \\
\hline Intracranial & $43(7.2 \%)$ & $40(8.5 \%)$ & $3(2.4 \%)$ & 0.018 \\
\hline Primary & $133(22.4 \%)$ & $107(22.9 \%)$ & $26(20.6 \%)$ & 0.594 \\
\hline Others $^{\mathrm{a}}$ & $90(15.2 \%)$ & $73(15.6 \%)$ & $17(13.5 \%)$ & 0.552 \\
\hline \multicolumn{5}{|l|}{ Antibiotic resistance of $\mathrm{AB}^{\mathrm{b}}$} \\
\hline Amikacin $(330 \text { vs. } 81)^{\mathrm{c}}$ & $145(24.4 \%)$ & $115(24.6 \%)$ & $30(23.8 \%)$ & 0.380 \\
\hline Ciprofloxacin (467 vs. 126$)^{\mathrm{c}}$ & $545(91.8 \%)$ & $431(92.1 \%)$ & $114(90.5 \%)$ & 0.701 \\
\hline Ceftazidime (463 vs. 125$)^{\mathrm{c}}$ & $556(93.6 \%)$ & $440(94 \%)$ & $116(92.1 \%)$ & 0.598 \\
\hline Tobramycin $(460 \text { vs. } 121)^{c}$ & $409(68.9 \%)$ & $316(67.5 \%)$ & $93(73.8 \%)$ & 0.068 \\
\hline Levofloxacin $(467 \text { vs. } 126)^{c}$ & $519(87.4 \%)$ & $412(88 \%)$ & $107(84.9 \%)$ & 0.532 \\
\hline Nitrofurantoin (431 vs. 117$)^{\mathrm{c}}$ & $541(91.1 \%)$ & $425(90.8 \%)$ & $116(92.1 \%)$ & 0.864 \\
\hline Cefoperazone/sulbactam $(460 \text { vs. } 125)^{c}$ & $520(87.5 \%)$ & $409(87.4 \%)$ & $111(88.1 \%)$ & 0.756 \\
\hline Gentamicin $(455 \text { vs. } 121)^{c}$ & $461(77.6 \%)$ & $358(76.5 \%)$ & $103(81.7 \%)$ & 0.229 \\
\hline Piperacillin/tazobactam $(241 \text { vs. } 61)^{c}$ & $273(46 \%)$ & $217(46.4 \%)$ & $56(44.4 \%)$ & 0.761 \\
\hline Carbapenems (467 vs. 126$)^{c}$ & $550(92.6 \%)$ & $435(92.9 \%)$ & $115(91.3 \%)$ & 0.673 \\
\hline Tigecycline (391 vs. 106$)^{c}$ & $165(27.8 \%)$ & $135(28.8 \%)$ & $30(23.8 \%)$ & 0.475 \\
\hline Colistin $(253 \text { vs. } 57)^{\mathrm{c}}$ & $9(1.5 \%)$ & $9(1.9 \%)$ & $0(0 \%)$ & 0.083 \\
\hline \multicolumn{5}{|l|}{ Treatment after the onset of BSIs } \\
\hline Appropriate empiric antibiotic treatment & $169(28.5 \%)$ & $120(25.6 \%)$ & $49(38.9 \%)$ & 0.003 \\
\hline
\end{tabular}

${ }^{\mathrm{a}}$ Biliary tract, heart surgery, urinary tract, and intraabdominal. ${ }^{\mathrm{b}} \mathrm{AB}$ : Acinetobacter baumannii; not all agents listed tested in all isolates. ${ }^{\mathrm{c}}$ The numbers in parentheses represent the total numbers of $\mathrm{AB}$ isolates that were subjected to susceptibility testing. 


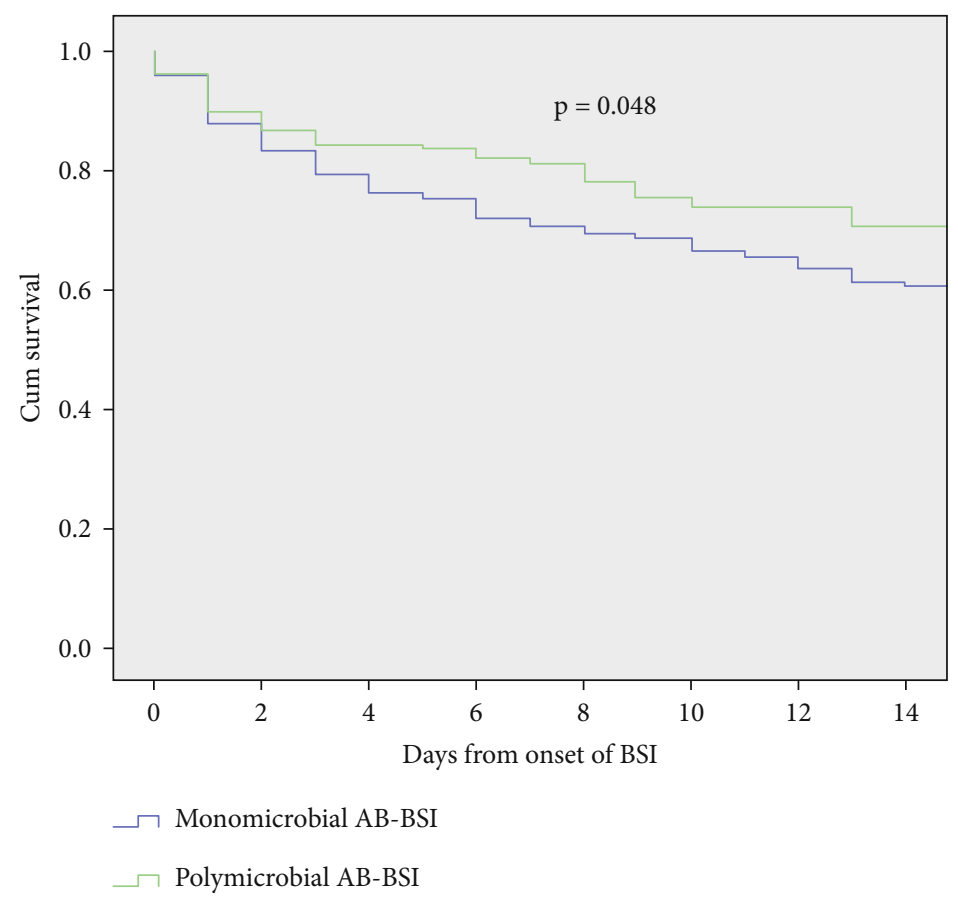

FIGURE 3: Kaplan-Meier estimates of 14-day survival in patients with polymicrobial AB-BSI and monomicrobial AB-BSI; Abbreviations: ABBSI: Acinetobacter baumannii bloodstream infection.

TABLE 5: Comparisons of outcomes between monomicrobial AB-BSIs and polymicrobial AB-BSIs.

\begin{tabular}{|c|c|c|c|c|}
\hline Prognostic indicators & Total & Monomicrobial & Polymicrobial & $p$ value \\
\hline Total hospitalization days (M) (IQR) & $29(18,50)$ & $27(16,45)$ & $40(21,68)$ & $<0.001$ \\
\hline Hospitalization days (M) after BSI (IQR) & $14(5,32)$ & $13(4,28)$ & $22(8,50)$ & $<0.001$ \\
\hline Total ICU residence days (M) (IQR) & $20(12,35)$ & $25(14,49)$ & $20(12,32)$ & 0.003 \\
\hline ICU residence days after BSI (M) (IQR) & $9(3,20)$ & $11(3,33)$ & $8(3,18)$ & 0.003 \\
\hline Sepsis $(n, \%)$ & $573(96.5 \%)$ & $454(97 \%)$ & $119(94.4 \%)$ & 0.167 \\
\hline Septic shock $(n, \%)$ & $167(28.1 \%)$ & $135(28.8 \%)$ & $32(25.4 \%)$ & 0.445 \\
\hline 14 -day mortality $(n, \%)$ & $211(35.5 \%)$ & $175(37.4 \%)$ & $36(28.6 \%)$ & 0.066 \\
\hline 28 -day mortality $(n, \%)$ & $252(42.4 \%)$ & $206(44 \%)$ & $46(36.5 \%)$ & 0.130 \\
\hline In-hospital mortality $(n, \%)$ & $283(47.6 \%)$ & $228(48.7 \%)$ & $55(43.7 \%)$ & 0.312 \\
\hline
\end{tabular}

demonstrate differences in mortality between patients with polymicrobial and monomicrobial AB-BSIs [15]. Furthermore, many variables (age, certain comorbidities, appropriate empirical therapy, and source of bacteraemia) were significantly different between polymicrobial and monomicrobial infection patients, limiting the interpretation of comparisons of the two groups.

This study had several limitations: (1) a retrospective design; (2) a small sample size (insufficient power to demonstrate difference in mortality) [15]; (3) a lack of antimicrobial susceptibility testing in all $\mathrm{AB}$ isolates; the use of two different antimicrobials susceptibility tests, different specific definitions of AB-BSI, and differences in characteristics between the two groups (polymicrobial vs. monomicrobial groups); (4) heterogeneous polymicrobial infections (the outcomes may differ depending on copathogens and/or sources of bacteraemia) [15]; (5) assessment of all-cause mortality rather than mortality attributable to infection; and (6) lack of consideration of in-hospital mortality and post-BSI length of stay as competing events in the statistical analysis [11]. Taken together, our findings might not be applicable to some other conditions; thus, future multicenter prospective studies are needed.

\section{Conclusion}

Approximately one-fifth of all AB-BSIs were polymicrobial in this cohort. The most common sources of polymicrobial AB-BSI were skin and soft tissue infections, and burn injury was the only independent risk factor for polymicrobial ABBSI. The post-BSI length of stay was significantly longer in polymicrobial infections patients, but other outcomes (including 14-day, 28-day, and in-hospital mortality) did not differ significantly between the groups. However, 
considering the limitations of this study, further studies are required to assess the impact of polymicrobial (vs. monomicrobial) AB-BSI on outcomes.

\section{Data Availability}

Data can be obtained from the corresponding author.

\section{Ethical Approval}

The study data comprised anonymized data from medical records collected as part of routine patient care, and the study received human research ethics approval (No.2019116) from the Ethics Committee of the Second Affiliated Hospital, Zhejiang University School of Medicine. Due to the retrospective nature of the study, the Ethics Committee determined that no patient consent was required.

\section{Disclosure}

We declare that this article is original work and has been published on the Research Square preprint platform before submission [38].

\section{Conflicts of Interest}

The authors declare that there are no conflicts of interest in this work.

\section{Authors' Contributions}

Zhenhua Qian and Shufang Zhang conceived the idea, performed the analysis, and drafted the manuscript. $\mathrm{Na} \mathrm{Li}$ and Weixing $\mathrm{Ma}$ interpreted the results and helped to revise the manuscript. Kai Zhang, Feizhen Song, Cheng Zheng, and Li Zhong helped collect data. Yesong Wang, Jiachang Cai, Hongwei Zhou, and Wei Cui helped analyse the data. Gensheng Zhang helped to frame the idea of the study and helped to analyse the data. All authors read and approved the final manuscript. Zhenhua Qian, Shufang Zhang, $\mathrm{Na}$ $\mathrm{Li}$, and Weixing Ma contributed equally to this work.

\section{Acknowledgments}

This work was supported in part by grants from the Natural Science Foundation of Zhejiang Province (No. LY19H150007, GS Zhang) and Medical and Health Research Programs of Zhejiang Province (Nos. 2018KY094 and 2019RC038, SF Zhang).

\section{References}

[1] M. Goto and M. N. Al-Hasan, "Overall burden of bloodstream infection and nosocomial bloodstream infection in North America and Europe," Clinical Microbiology and Infection, vol. 19, pp. 501-509, 2013.

[2] S. Fengcai, X. Di, H. Qianpeng, Z. Hongke, and D. Yiyu, "Microbial characteristics in culture-positive sepsis and risk factors of polymicrobial infection in ICU," Zhonghua wei Zhong Bing ji jiu yi xue, vol. 27, pp. 718-723, 2015.
[3] X. Wang, C. Zhao, H. Li et al., "Microbiological profiles of pathogens causing nosocomial bacteremia in 2011, 2013 and 2016," Chinese Journal of Biotechnology, vol. 34, no. 8, pp. 1205-1217, 2018.

[4] A. C. Gales, M. Castanheira, R. N. Jones, and H. S. Sader, "Antimicrobial resistance among gram-negative bacilli isolated from Latin America: results from SENTRY Antimicrobial Surveillance Program (Latin America, 2008-2010)," vol. 73, pp. 354-360, 2012.

[5] P. Kiratisin, A. Chongthaleong, T. Y. Tan et al., "Comparative in vitro activity of carbapenems against major gram-negative pathogens: results of Asia-Pacific surveillance from the COMPACT II study," International Journal of Antimicrobial Agents, vol. 39, pp. 311-316, 2012.

[6] J. M. Cisneros and J. Rodríguez-Baño, "Nosocomial bacteremia due to Acinetobacter baumannii: epidemiology, clinical features and treatment," Clinical Microbiology and Infection, vol. 8, pp. 687-693, 2002.

[7] G. Metan, F. Sariguzel, and B. Sumerkan, "Factors influencing survival in patients with multi-drug-resistant Acinetobacter bacteraemia," European Journal of Internal Medicine, vol. 20, pp. 540-544, 2009.

[8] A. Nutman, R. Glick, E. Temkin et al., "A case-control study to identify predictors of 14-day mortality following carbapenemresistant Acinetobacter baumannii bacteraemia," Clinical Microbiology and Infection, vol. 20, 2015.

[9] Z. Gu, Y. Han, T. Meng et al., "Risk factors and clinical outcomes for patients with Acinetobacter baumannii bacteremia," Medicine, vol. 95, 2016.

[10] H. Wisplinghoff, W. Perbix, and H. Seifert, "Risk factors for nosocomial bloodstream infections due to Acinetobacter baumannii: a case-control study of adult burn patients," Clinical Infectious Diseases, vol. 28, pp. 59-66, 1999.

[11] S. Karakonstantis, A. Gikas, E. Astrinaki, and E. I. Kritsotakis, "Excess mortality due to pandrug-resistant Acinetobacter baumannii infections in hospitalized patients," Journal of Hospital Infection, vol. 106, pp. 447-453, 2020.

[12] S. Karakonstantis, E. I. Kritsotakis, and A. Gikas, "Pandrugresistant gram-negative bacteria: a systematic review of current epidemiology, prognosis and treatment options," vol. 75, pp. 271-282, 2020.

[13] A. Tabah, D. Koulenti, K. Laupland et al., "Characteristics and determinants of outcome of hospital-acquired bloodstream infections in intensive care units: the EUROBACT International Cohort Study," Intensive Care Medicine, vol. 38, pp. 1930-1945, 2012.

[14] H. Wisplinghoff, T. Bischoff, S. M. Tallent, H. Seifert, R. P. Wenzel, and M. B. Edmond, "Nosocomial bloodstream infections in US hospitals: analysis of 24,179 cases from a prospective nationwide surveillance study," Clinical infectious diseases, vol. 39, pp. 309-317, 2004.

[15] S. Karakonstantis and E. I. Kritsotakis, "Systematic review and meta-analysis of the proportion and associated mortality of polymicrobial (vs monomicrobial) pulmonary and bloodstream infections by Acinetobacter baumannii complex," Infection, vol. 49, no. 6, pp. 1149-1161, 2021.

[16] M. Pavlaki, G. Poulakou, P. Drimousis et al., "Polymicrobial bloodstream infections: epidemiology and impact on mortality," Journal of Global Antimicrobial Resistance, vol. 1, no. 4, pp. 207-212, 2013. 
[17] C. Zheng, S. Zhang, Q. Chen et al., "Clinical characteristics and risk factors of polymicrobial Staphylococcus aureus bloodstream infections," Antimicrobial Resistance \& Infection Control, vol. 9, no. 1, 2020.

[18] C. Zheng, J. Cai, H. Liu et al., "Clinical characteristics and risk factors in mixed-enterococcal bloodstream infections," Infection and Drug Resistance, vol. 12, pp. 3397-3407, 2019.

[19] F. Song, K. Zhang, J. Huang et al., "Clinical characteristics, risk factors, and outcomes of patients with polymicrobial Klebsiella pneumoniae bloodstream infections," BioMed Research International, vol. 2021, Article ID 6619911, 10 pages, 2021.

[20] L. Zhong, S. Zhang, K. Tang et al., "Clinical characteristics, risk factors and outcomes of mixed Candida albicans/bacterial bloodstream infections," BMC Infectious Diseases, vol. 20, 2020.

[21] Y. C. Wang, W. W. Ku, Y. S. Yang et al., "Is polymicrobial bacteremia an independent risk factor for mortality in Acinetobacter baumannii bacteremia?," Journal of Clinical Medicine, vol. 9, no. 1, 2020.

[22] T. C. Horan, M. Andrus, and M. A. Dudeck, "CDC/NHSN surveillance definition of health care-associated infection and criteria for specific types of infections in the acute care setting," American Journal of Infection Control, vol. 36, no. 5, pp. 309-332, 2008.

[23] R. M. Humphries, J. Ambler, S. L. Mitchell et al., "CLSI methods development and standardization working group best practices for evaluation of antimicrobial susceptibility tests," Journal of Clinical Microbiology, vol. 56, 2018.

[24] M. Singer, C. S. Deutschman, C. W. Seymour et al., "The third international consensus definitions for sepsis and septic shock (sepsis-3)," JAMA, vol. 315, no. 8, pp. 801-810, 2016.

[25] J. L. García-Garmendia, C. Ortiz-Leyba, J. Garnacho-Montero et al., "Risk factors for Acinetobacter baumannii nosocomial bacteremia in critically ill patients: a cohort study," Clinical Infectious Diseases, vol. 33, no. 7, pp. 939-946, 2001.

[26] J. D. Sun, S. F. Huang, S. S. Yang, S. L. Pu, C. M. Zhang, and L. P. Zhang, "Impact of carbapenem heteroresistance among clinical isolates of invasive Escherichia coli in Chongqing, Southwestern China," Clinical Microbiology and Infection, vol. 21, 2015.

[27] M. R. Halachev, J. Z. M. Chan, C. I. Constantinidou et al., "Genomic epidemiology of a protracted hospital outbreak caused by multidrug-resistant Acinetobacter baumannii in Birmingham, England," Genome Medicine, vol. 6, no. 11, 2014.

[28] L. Aliaga, J. D. Mediavilla, J. Llosa, C. Miranda, and M. RosaFraile, "Clinical significance of polymicrobial versus monomicrobial bacteremia involving Pseudomonas aeruginosa," European Journal of Clinical Microbiology and Infectious Diseases, vol. 19, pp. 871-874, 2000.

[29] Q. Liu, J. Wu, Z. Wang, X. Wu, G. Wang, and J. Ren, "Polymicrobial bacteremia involving Klebsiella pneumoniae in patients with complicated intra-abdominal infections: Frequency, copathogens, risk factors, and clinical outcomes," Surgical Infections, vol. 20, pp. 317-325, 2019.

[30] N.-Y. Lee, H.-C. Lee, N.-Y. Ko et al., "Clinical and economic impact of multidrug resistance in nosocomial Acinetobacter baumannii bacteremia," Infection Control \& Hospital Epidemiology, vol. 28, pp. 713-719, 2007.

[31] E. A. Idelevich, H. Seifert, M. Sundqvist et al., "Microbiological diagnostics of bloodstream infections in Europe-an ESGBIES survey," Clinical Microbiology and Infection, vol. 25, no. 11, pp. 1399-1407, 2019.

[32] A. Tabah, N. Buetti, F. Barbier, and J. F. Timsit, "Current opinion in management of septic shock due to gram-negative bacteria," Current Opinion in Infectious diseases, vol. 34, pp. 718727, 2021

[33] S. Comini, G. Bianco, M. Boattini et al., "Evaluation of the Amplex eazyplex SuperBug Acineto test for direct detection of multi-drug resistant Acinetobacter baumannii bloodstream infections in high endemicity settings," Journal of Hospital Infection, vol. 117, pp. 179-181, 2021.

[34] C. Q. Tang, J. Q. Li, B. M. Shou et al., "Epidemiology and outcomes of bloodstream infections in 177 severe burn patients from an industrial disaster: a multicentre retrospective study," Clinical Microbiology and Infection, vol. 24, 2018.

[35] D. Church, S. Elsayed, O. Reid, B. Winston, and R. Lindsay, "Burn wound infections," Clinical Microbiology Reviews, vol. 19, no. 2, pp. 403-434, 2006.

[36] S. G. Santucci, S. Gobara, C. R. Santos, C. Fontana, and A. S. Levin, "Infections in a burn intensive care unit: experience of seven years," The Journal of Hospital Infection, vol. 53, no. 1, pp. 6-13, 2003.

[37] J. Fitzwater, G. F. Purdue, J. L. Hunt, and G. E. O’Keefe, “The risk factors and time course of sepsis and organ dysfunction after burn trauma," Journal of Trauma and Acute Care Surgery, vol. 54, pp. 959-966, 2003.

[38] Z. Qian, S. Zhang, N. Li et al., "Risk Factors and Clinical Outcomes of Mixed Acinetobacter Baumannii Bloodstream Infection," 2021. 